\title{
Vector imaging of electric field-induced reversible magnetization reversal in exchange-biased multiferroic heterostructures
}

\author{
Xinger Zhao ${ }^{1}$, Zhongqiang $\mathrm{Hu}^{1 *}$, Ting Fang ${ }^{1}$, Yuxin Cheng ${ }^{1}$, Keqing Shi ${ }^{2}$, Yi-Xin Weng ${ }^{3}$, Yongjun $\mathrm{Du}^{1}$, \\ Jingen $\mathrm{Wu}^{1}$, Mengmeng Guan ${ }^{1}$, Zhiguang Wang ${ }^{1}$, Ziyao Zhou ${ }^{1}$, Ming Liu ${ }^{1}$ and Jing-Ye Pan ${ }^{3 *}$
}

\begin{abstract}
Exchange bias between ferromagnetic and antiferromagnetic layers has been widely utilized in spintronic devices. Controlling the exchange bias in magnetic multilayers by an electric field (E-field) has been proposed as a low-power solution for manipulating the macroscopic properties such as exchange bias fields and magnetization values, while how the magnetic domains respond to the E-fields has rarely been reported in an exchange-biased system. Here, we realize the vector imaging of reversible electrical modulation of magnetization reversal in exchange-biased $\mathrm{CoFeB} / \mathrm{IrMn} / \mathrm{PMN}-\mathrm{PT}$ (011) multiferroic heterostructures, utilizing in-situ quantitative magneto-optical Kerr effect (MOKE) microscopy. Under the electrical control, magnetic domains at -80 Oe rotate reversibly between around $160^{\circ}$ and $80^{\circ}-120^{\circ}$, whose transverse components reverse from $225^{\circ}$ to $45^{\circ}$ correspondingly. Moreover, pixel-by-pixel comparisons are conducted to further imply the reversible magnetization reversal by E-fields. Efield-induced reversible magnetization reversal is also demonstrated without applying external magnetic fields. Vector imaging of electrical manipulation of exchange bias is of great significance in understanding the magnetoelectric effect and the development of next-generation spintronic devices.
\end{abstract}

Keywords: exchange bias, magnetic domains, multiferroics, magnetization reversal

\section{INTRODUCTION}

Exchange bias, the interfacial coupling between ferromagnetic and antiferromagnetic materials, is of great significance in spintronic devices, such as memories and sensors [1-6], due to the ability to stabilize magnetization in spin-value structures [7]. And sensors based on exchange bias also have great potential as an imaging modality in biomedical applications, such as detection and analysis of magnetic nanoparticles $[8,9]$. In pursuit of lower power consumption, electric fields (E-fields), instead of electric currents, have been proposed as an energy-efficient alternative to modulate exchange bias [10-15]. Reversible E-field control between the positive and negative exchange bias field $\left(H_{\mathrm{EB}}\right)$ was realized in the $(\mathrm{Pd} / \mathrm{Co})_{3} / \mathrm{Pd} / \mathrm{Cr}_{2} \mathrm{O}_{3}$ and $\mathrm{LaSrMnO}_{3} /$ $\mathrm{BiFeO}_{3}$ heterostructures [16,17]. And the obvious and negligible exchange bias states could also be switched by E-fields via resistive switching of the $\mathrm{NiO}$ layer in $\mathrm{Co} / \mathrm{NiO}$ devices [18]. Moreover, E-field regulation of magnetization reversal has been acquired in exchange-biased multiferroic heterostructures [19-22]. It was demonstrated that near $180^{\circ}$ irreversible switching of magnetization was induced by E-fields in FeMn/ $\mathrm{NiFe} / \mathrm{FeGaB}$ multilayers deposited on the lead zinc niobate-lead titanate (PZN-PT) (011) substrates [21]. Reversible control of magnetization reversal was further obtained in $\mathrm{CoFeB} / \mathrm{IrMn} /$ $\mathrm{Pb}\left(\mathrm{Mg}_{1 / 3} \mathrm{Nb}_{2 / 3}\right)_{0.7} \mathrm{Ti}_{0.3} \mathrm{O}_{3}$ (PMN-PT) (011) heterostructures for only one magnetization value at the selected identical magnetic field ( $\mathrm{H}$-field) under each of different $\mathrm{E}$-fields as a result of the separated hysteresis regions of loops [22].

E-field control of macroscopic properties, like $H_{\mathrm{EB}}$ and magnetization values, has been extensively studied, but the magnetic domain evolution under E-fields in exchange-biased systems is still unclear. Relying on the vector imaging techniques, electrical regulation of magnetic domains has been achieved in many different forms [23-26], such as realizing various E-field responses of magnetic domains in CoFeB/PMN-PT (001) heterostructures attributed to different ferroelectric domain switching $[27,28]$, less than $90^{\circ}$ rotation of domains via E-fieldinduced shear strain effect $[29,30]$, and reversible domain-wall motion based on the elastic coupling between magnetic and ferroelectric domain walls [31,32], etc. Meanwhile, E-fields can also be utilized to manipulate the domain wall velocity $[33,34]$. However, vector imaging of magnetic domain evolution with Efields has been rarely investigated in exchange-biased multiferroic heterostructures.

In this work, we report the vector imaging of reversible E-field control of magnetization reversal in exchange-biased $\mathrm{CoFeB} /$ IrMn/PMN-PT(011) multiferroic heterostructures via in-situ quantitative magneto-optical Kerr effect (MOKE) microscopy. At $-80 \mathrm{Oe}$, electrical modulation induces deterministic and repeatable rotation of the magnetic domains between approximately $160^{\circ}$ and $80^{\circ}-120^{\circ}$, accompanied by the reversible magnetization reversal. Furthermore, the pixel-by-pixel comparisons of magnetic domains are implemented to confirm the reversibility and reproducibility further. Additionally, E-field regulation of magnetic domain evolution can also be achieved at $0 \mathrm{Oe}$ via selecting the annealing direction reasonably. Vector imaging

\footnotetext{
${ }^{1}$ Electronic Materials Research Laboratory, Key Laboratory of the Ministry of Education \& International Center for Dielectric Research, School of Electronic Science and Engineering, State Key Laboratory for Mechanical Behavior of Materials, Xi'an Jiaotong University, Xi'an 710049, China

2 Translational Medicine Laboratory, the First Affiliated Hospital of Wenzhou Medical University, Wenzhou 325000, China

${ }^{3}$ Key Laboratory of Intelligent Critical Care and Life Support Research of Zhejiang Province, Department of Intensive Care, the First Affiliated Hospital of Wenzhou Medical University, Wenzhou 325000, China

*Corresponding authors (emails: zhongqianghu@xjtu.edu.cn (Hu Z); panjingye@wzhospital.cn (Pan JY))
} 
of magnetization reversal constitutes an important step towards the study of exchange bias and magnetoelectric effect.

\section{EXPERIMENTAL SECTION}

\section{Sample preparation}

Multilayer films with the structure of $\mathrm{Ta}(3 \mathrm{~nm}) / \mathrm{CoFeB}(20 \mathrm{~nm}) /$ $\operatorname{IrMn}(8 \mathrm{~nm}) / \mathrm{NiFe}(2 \mathrm{~nm}) / \mathrm{Ta}(5 \mathrm{~nm})$ were deposited on the unpolarized PMN-PT(011) piezoelectric single-crystal substrates via direct-current magnetron sputtering with a base pressure below $1.5 \times 10^{-7}$ Torr $(1$ Torr $=133.3 \mathrm{~Pa})$ at room temperature. The 2-nm NiFe layer was prepared to assist the formation of [111]-oriented IrMn layer to induce exchange coupling between the IrMn and $\mathrm{CoFeB}$ layers. The back of the substrates was covered by a $\mathrm{Cu}(20 \mathrm{~nm}) / \mathrm{Ta}(5 \mathrm{~nm})$ bilayer as the bottom electrode. To enhance the exchange bias, the deposited samples were annealed at $280^{\circ} \mathrm{C}$ for $30 \mathrm{~min}$ with an $\mathrm{H}$-field of $3000 \mathrm{Oe}$.

\section{Experiment characterization}

In-situ magnetic domain imaging under $\mathrm{H}$-fields and/or E-fields was conducted by a quantitative MOKE microscope (Evico Magnetics, em-Kerr-Highres) in longitudinal mode. Its 4-pole horizontal magnet and rotatable displacement table contribute well to the investigation of the angular dependence of exchange bias. Commercial software with the MOKE system was utilized to help analyze the magnetization directions of magnetic domains with a deviation of less than $5^{\circ}$. For each test, calibration functions need to be measured firstly, and then the magnetization directions can be acquired by substituting desired magnetic domain patterns into the functions. Magnetic hysteresis (M-H) loops were also measured by MOKE. In addition, E- fields were applied using a Keithley 6517B Electrometer through the thickness direction of the PMN-PT substrates.

\section{RESULTS AND DISCUSSION}

To investigate E-field modulation of magnetic domain evolution in exchange-biased systems, $\mathrm{Ta}(3 \mathrm{~nm}) / \mathrm{CoFeB}(20 \mathrm{~nm}) / \mathrm{IrMn}$ $(8 \mathrm{~nm}) / \mathrm{NiFe}(2 \mathrm{~nm}) / \mathrm{Ta}(5 \mathrm{~nm})$ multilayers were deposited on the 0.5 -mm-thick piezoelectric crystals PMN-PT(011) by magnetron sputtering at room temperature. The inserted 2-nm NiFe film played an important role in inducing crystallization of IrMn along the [111] direction. To enhance the exchange coupling between the IrMn and CoFeB layers, the prepared samples were annealed at $280^{\circ} \mathrm{C}$ for $30 \mathrm{~min}$ with the annealing $\mathrm{H}$-field $\left(H_{\text {anneal }}\right)$ along the [01-1] direction.

Fig. 1a shows the measurement configuration of the exchangebiased multilayers. E-fields were applied through the thickness direction of the PMN-PT substrates, and the heterostructures were polarized with an E-field of $10 \mathrm{kV} \mathrm{cm}^{-1}$ before tests. $\theta$ is defined as the direction of $\mathrm{H}$-fields where the [100] and [01-1] direction are set as $\theta=0^{\circ}$ and $\theta=90^{\circ}$, respectively. Angular dependence of exchange bias was investigated with an interval of $15^{\circ}$, and $\mathrm{M}-\mathrm{H}$ loops at $\theta=0^{\circ}, 45^{\circ}, 90^{\circ}$ can be found in Fig. $1 \mathrm{~b}$, indicating the strong dependence of $H_{\mathrm{EB}}$ on $\theta$. The normalized gray level values are equivalent to the normalized magnetization $\left(M / M_{\mathrm{s}}\right)$.

Fig. 1c illustrates the dynamic magnetization reversal process with the $\mathrm{H}$-field ascended from -300 to $300 \mathrm{Oe}$ at $\theta=45^{\circ}$. To simplify the description of the magnetization directions, angles anticlockwise rotating from $0^{\circ}$ to $360^{\circ}$ are utilized to indicate the relative orientation of magnetizations, as shown around the color wheel on the left of Fig. 1c. In detail, $0^{\circ}$ corresponds to the
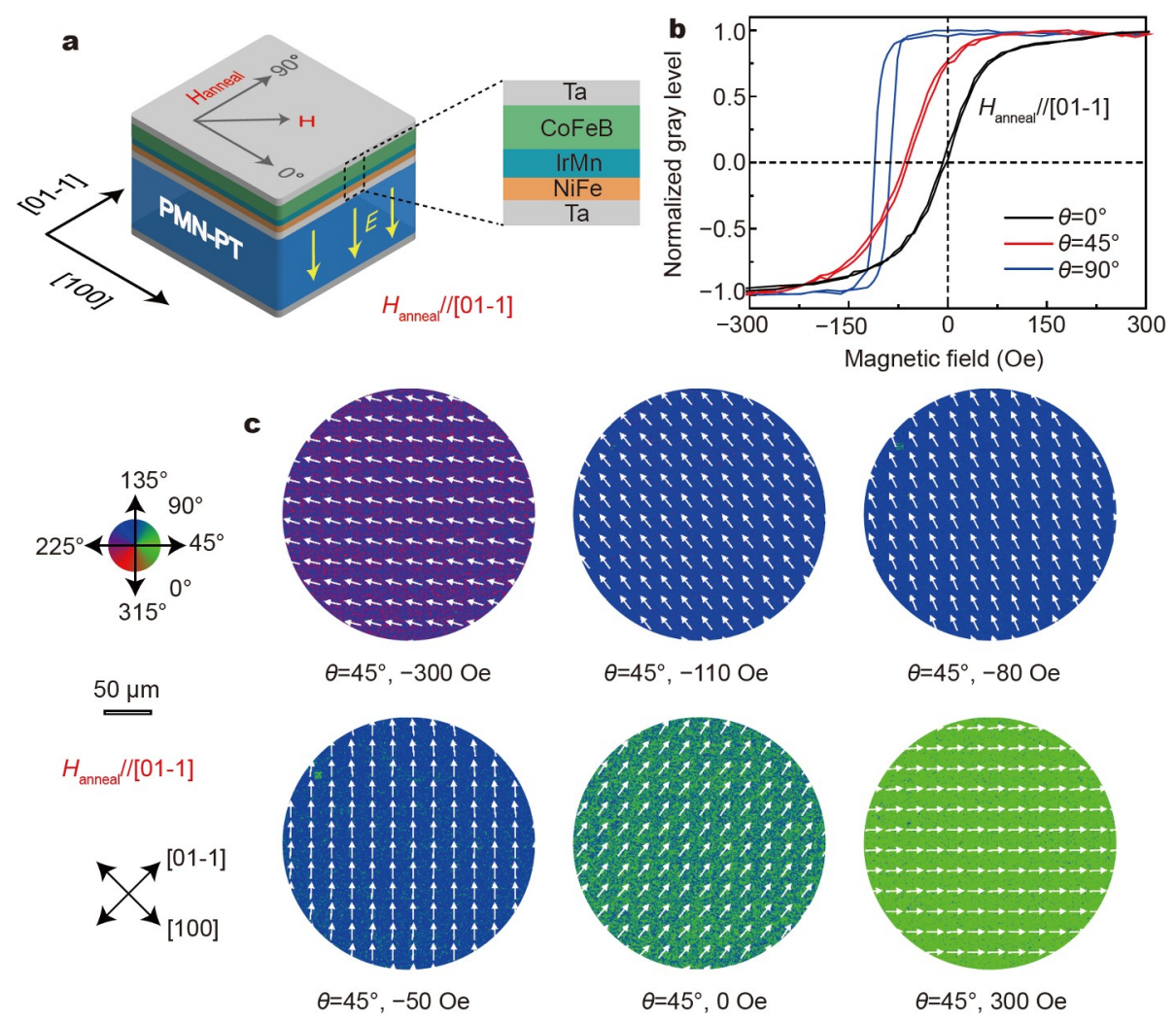

Figure 1 (a) Schematic of CoFeB/IrMn/PMN-PT(011) exchange-biased multiferroic heterostructure. (b) Angular dependence of M-H loops. (c) Magnetic domain patterns during a magnetization reversal process at $\theta=45^{\circ}$. The color wheel and scale bar are shown on the left. 
[100] direction, and $90^{\circ}$ is the [01-1] direction. The magnetization directions of magnetic domains rotate gradually from approximately $225^{\circ}$ to $180^{\circ}$ when the $\mathrm{H}$-field is increased from -300 to $-110 \mathrm{Oe}$. At $-50 \mathrm{Oe}$, the magnetization directions are parallel to $135^{\circ}$. With the $\mathrm{H}$-field increased to $0 \mathrm{Oe}$, that is, no external $\mathrm{H}$-field applied, magnetic domains in the field of vision follow $90^{\circ}$, which is consistent with $H_{\text {anneal }}$ facilitating the easy axis in the [01-1] direction. As the $\mathrm{H}$-field continues to increase, the magnetization directions progressively rotate to $45^{\circ}$, which is the direction of the external $\mathrm{H}$-field. Domains at $\theta=0^{\circ}$ and $\theta=$ $90^{\circ}$ can be found in Figs S1 and S2.

The magnetization reversal processes at different E-fields were investigated with $\mathrm{H}$-fields at $\theta=45^{\circ}$ from -300 to $300 \mathrm{Oe}$, and the partial processes from -120 to $-50 \mathrm{Oe}$ are illustrated in Fig. $2 \mathrm{a}-\mathrm{c}$. Fig. $2 \mathrm{~d}$ shows the corresponding $\mathrm{M}-\mathrm{H}$ loops at various E-fields. Despite the obvious differences among the $\mathrm{M}-\mathrm{H}$ loops, there is a likeness between the magnetization processes at 0 $4 \mathrm{kV} \mathrm{cm}^{-1}$, where the magnetization reversal is accomplished by the clockwise rotation of magnetic domains. It is noteworthy that the $\mathrm{H}$-fields required for magnetic domains in certain directions are driven to decrease with the increase of E-fields.
For instance, the magnetization directions of magnetic domains do not rotate to $135^{\circ}$ until the $\mathrm{H}$-field is ascended to $-50 \mathrm{Oe}$ under $0 \mathrm{kV} \mathrm{cm}^{-1}$, but magnetic domains in this direction can be just acquired at -80 Oe under $2 \mathrm{kV} \mathrm{cm}^{-1}$ and at -110 Oe under $4 \mathrm{kV} \mathrm{cm}^{-1}$. Nevertheless, the magnetization process at $6 \mathrm{kV} \mathrm{cm}^{-1}$ is different from that at $0-4 \mathrm{kV} \mathrm{cm}^{-1}$ as mentioned above, where distinct magnetic domain wall motion can be observed around $-110 \mathrm{Oe}$, corresponding to the sharp rise of the $\mathrm{M}-\mathrm{H}$ loop from negative to positive values instead of smooth variation. Fig. $2 \mathrm{e}$ displays that $M / M_{\mathrm{s}}$ values increase with the rise of $\mathrm{H}$-fields, corresponding to the clockwise variation of magnetic domains under electrical modulation. Angular dependence of $\mathrm{M}-\mathrm{H}$ loops is shown in Fig. S3.

Fig. 3 illustrates electrical modulation of reversible magnetization reversal with the bias $\mathrm{H}$-field fixed at -80 Oe for $\theta=45^{\circ}$. The $\mathrm{H}$-field of -80 Oe was selected due to the only one magnetization distribution presented at each E-field from 0 to $6 \mathrm{kV} \mathrm{cm}^{-1}$, which is the necessary condition for the reversible control by E-fields alone. The bias $\mathrm{H}$-field at $-80 \mathrm{Oe}$ was increased from -300 Oe before tests, and it was kept constant during the electrical manipulation. Fig. 3a displays the magnetic

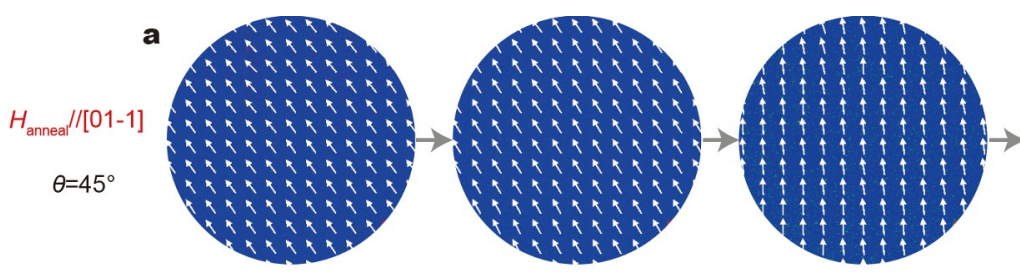

$2 \mathrm{kV} \mathrm{cm}^{-1},-120 \mathrm{Oe}$

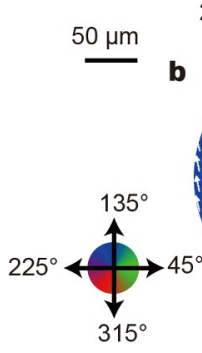

$2 \mathrm{kV} \mathrm{cm}^{-1},-80 \mathrm{Oe}$

$4 \mathrm{kV} \mathrm{cm}^{-1},-80 \mathrm{Oe}$
$4 \mathrm{kV} \mathrm{cm}^{-1},-120 \mathrm{Oe} \quad 4 \mathrm{kV} \mathrm{cm}^{-1},-110 \mathrm{Oe}$

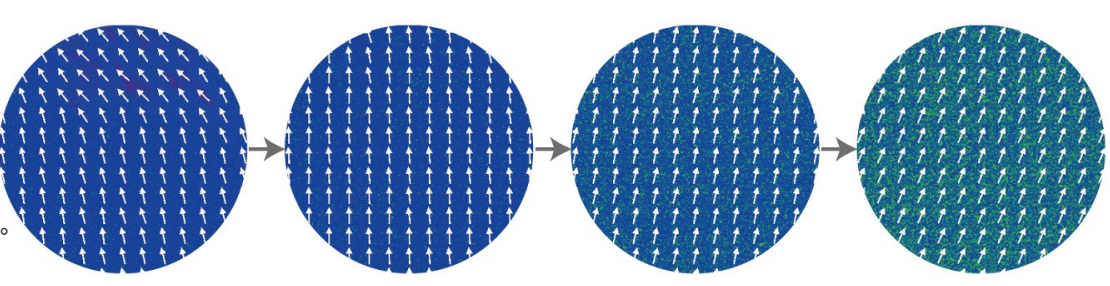

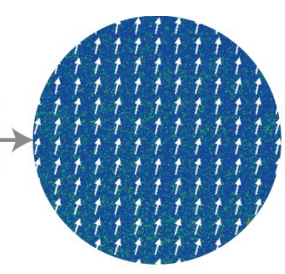

$2 \mathrm{kV} \mathrm{cm}^{-1},-50 \mathrm{Oe}$

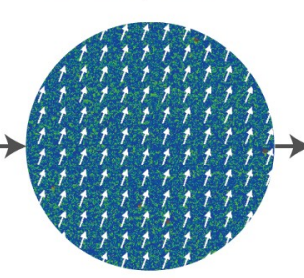

$6 \mathrm{kV} \mathrm{cm}^{-1},-80 \mathrm{Oe}$
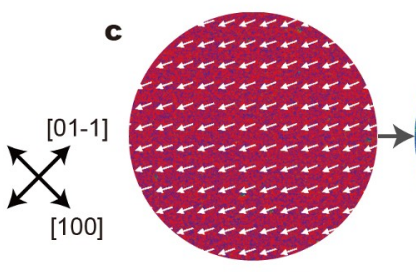

$6 \mathrm{kV} \mathrm{cm}^{-1},-120 \mathrm{Oe}$

$6 \mathrm{kV} \mathrm{cm}^{-1},-110 \mathrm{Oe}$
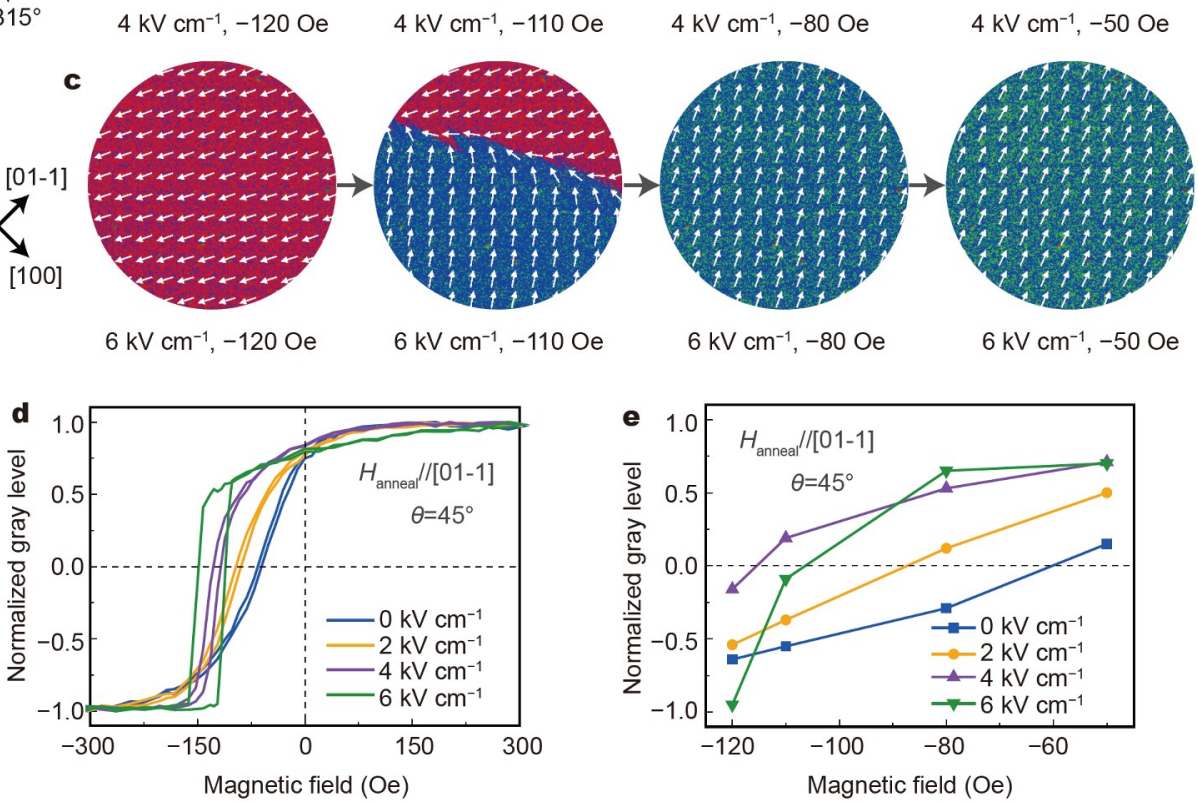

Figure 2 Magnetization processes and the corresponding M-H loops under various E-fields at $\theta=45^{\circ}$. (a-c) Magnetic domain evolution from -120 to $-50 \mathrm{Oe}$ at (a) $2 \mathrm{kV} \mathrm{cm}^{-1}$, (b) $4 \mathrm{kV} \mathrm{cm}^{-1}$ and (c) $6 \mathrm{kV} \mathrm{cm}^{-1}$, respectively. (d) E-field regulation of M-H loops. (e) Values of $M / M_{\mathrm{s}}$ as a function of $\mathrm{H}$-fields under various E-fields. 

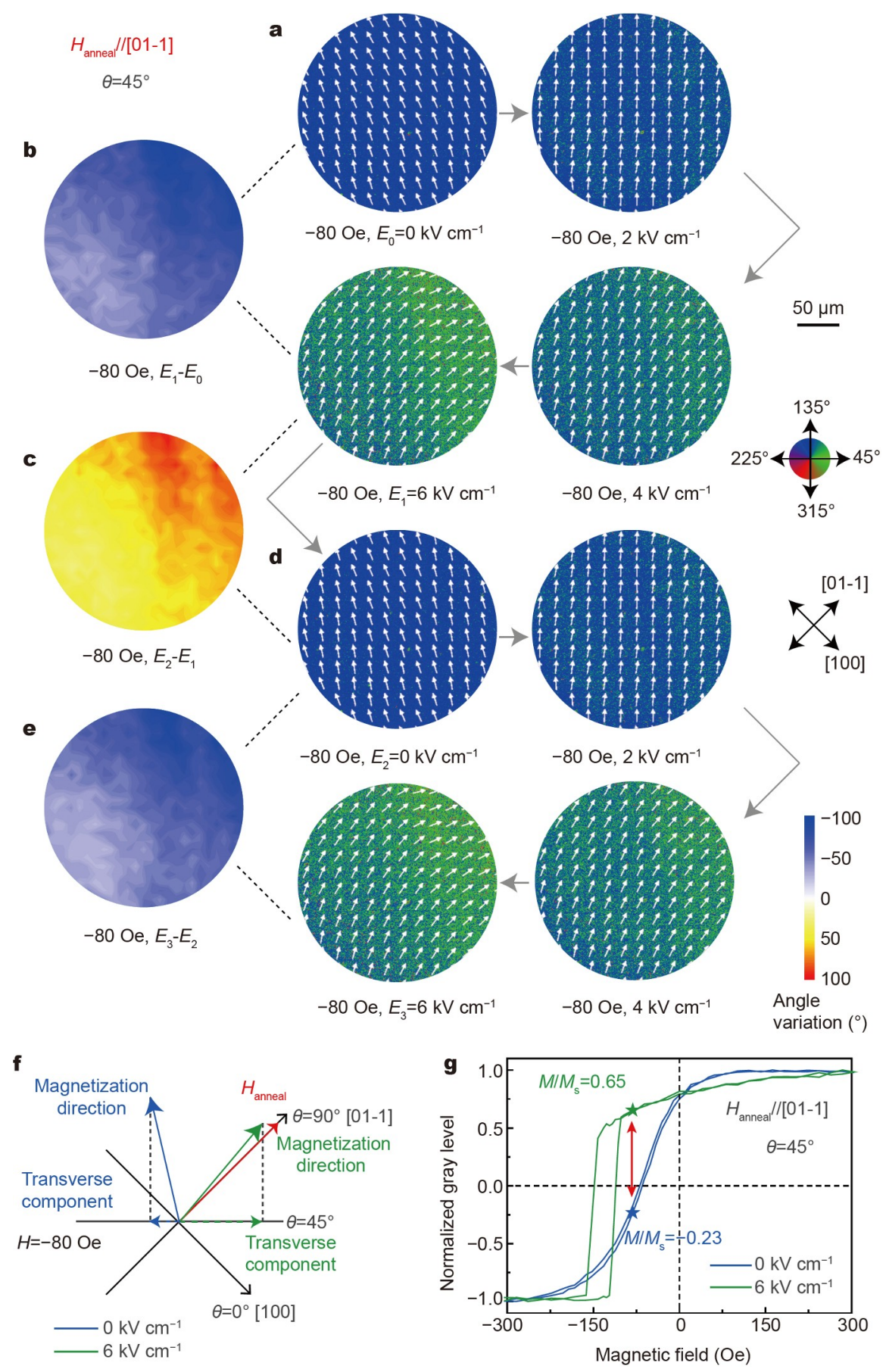

Figure 3 Reversible electrical control of magnetization reversal under -80 Oe at $\theta=45^{\circ}$. (a) Magnetic domains evolution under E-fields with magnetization reversal. (b, c) Pixel-by-pixel comparisons of magnetic domains at $E_{0}=0 \mathrm{kV} \mathrm{cm}^{-1}$ and $E_{1}=6 \mathrm{kV} \mathrm{cm}^{-1}$ (b), and at $E_{1}=6 \mathrm{kV} \mathrm{cm}^{-1}$ and $E_{2}=0 \mathrm{kV} \mathrm{cm}^{-1}$ (c), respectively. (d) Reproducibility of reversible magnetization reversal driven by E-fields solely with the bias $\mathrm{H}$-field unchanged. (e) Corresponding variation angles of domains at $E_{2}=0 \mathrm{kV} \mathrm{cm}^{-1}$ and $E_{3}=6 \mathrm{kV} \mathrm{cm}^{-1}$. (f) Sketch of electrical control of magnetization reversal. (g) M- $\mathrm{H}$ loops at 0 and $6 \mathrm{kV} \mathrm{cm}{ }^{-1}$. Stars represent the values of $M / M_{\mathrm{s}}$ at $-80 \mathrm{Oe}$.

domain evolution under the applied E-fields ranging from $E_{0}=$ $0 \mathrm{kV} \mathrm{cm}^{-1}$ to $E_{1}=6 \mathrm{kV} \mathrm{cm}^{-1}$, where electrical modulation of the magnetization reversal is realized with the transverse components of magnetizations driven from $225^{\circ}$ to $45^{\circ}$. In detail, as the E-field is ascended from 0 to $2 \mathrm{kV} \mathrm{cm}^{-1}$, the magnetic domains are urged to clockwise rotate to $135^{\circ}$ from approximately $160^{\circ}$. When the E-field continues to increase to $4 \mathrm{kV} \mathrm{cm}^{-1}$, magneti- zation directions are along $110^{\circ}-120^{\circ}$, and with the E-field of $6 \mathrm{kV} \mathrm{cm}^{-1}$, the magnetic domains are induced to mainly lying in $80^{\circ}-120^{\circ}$. Meanwhile, pixel-by-pixel comparisons of magnetic domains at various E-fields were performed to present the variation angles of domains induced by E-fields. Fig. 3b displays that the applied E-fields from $E_{0}=0 \mathrm{kV} \mathrm{cm}^{-1}$ to $E_{1}=6 \mathrm{kV} \mathrm{cm}^{-1}$ induce the variation angles between $-31^{\circ}$ and $-103^{\circ}$, and $97 \%$ 
are concentrated in the range of $-31^{\circ}$ to $-90^{\circ}$. In detail, the smaller angles from $-31^{\circ}$ to $-50^{\circ}$ are located in the left lower part of the field of view, accounting for $32 \%$, and the larger angles over $-70^{\circ}$ occupy $27 \%$ in the right upper.

Once the E-field is removed from $E_{1}=6 \mathrm{kV} \mathrm{cm}^{-1}$ to $E_{2}=$ $0 \mathrm{kV} \mathrm{cm}^{-1}$, the anticlockwise rotation driven by E-fields induces magnetic domains recovering the initial magnetization distribution of electrical modulation, with $96 \%$ variation angles from $31^{\circ}$ to $90^{\circ}$, as shown in Fig. $3 \mathrm{c}$. With the transverse components of domains switching back to $225^{\circ}$ from $45^{\circ}$, the reversible magnetization reversal is realized by electrical modulation alone. Moreover, the E-field-induced reversible magnetization reversal is reproducible, as illustrated in Fig. $3 \mathrm{~d}$. When the E-fields are applied once more, the magnetic domains are induced to clockwise rotate again from approximately $160^{\circ}$ at $E_{2}$ $=0 \mathrm{kV} \mathrm{cm}^{-1}$ to $80^{\circ}-120^{\circ}$ at $E_{3}=6 \mathrm{kV} \mathrm{cm}^{-1}$, with the transverse components reversing from $225^{\circ}$ to $45^{\circ}$. In other words, the reversible control of magnetization reversal can be achieved repeatedly by changing the E-fields solely. Fig. $3 e$ shows the variation angles between domains at $E_{2}=0 \mathrm{kV} \mathrm{cm}^{-1}$ and $E_{3}=$ $6 \mathrm{kV} \mathrm{cm}^{-1}$, where $94 \%$ are between $-31^{\circ}$ and $-90^{\circ}$, and variation angles less than $-50^{\circ}$ or over $-70^{\circ}$ are centered in the left lower or right upper with the proportion of $40 \%$ or $19 \%$.

For the case with $H_{\text {anneal }}$ along the [01-1] direction, the initial easy axis direction at $0 \mathrm{kV} \mathrm{cm}^{-1}$ is set along the [01-1] direction, and the applied E-fields result in that the [01-1] direction becomes easier to be magnetized. Thus, if there is no external $\mathrm{H}$ field applied, the magnetization directions of domains should be both around $90^{\circ}$ (the [01-1] direction) with the transverse components along $45^{\circ}$, that is, the electrical control of magnetization reversal cannot be realized at 0 Oe. The magnetic domain patterns at 0 Oe under $0 \mathrm{kV} \mathrm{cm}^{-1}$ can be found in Fig. $1 \mathrm{c}$. As shown in Fig. 3f, an appropriate $\mathrm{H}$-field can be selected to drag the transverse components of domains along $225^{\circ}$ at $0 \mathrm{kV} \mathrm{cm}^{-1}$ with that lying in $45^{\circ}$ under the E-field of $6 \mathrm{kV} \mathrm{cm}^{-1}$. Therefore, electrical regulation of magnetization reversal can be accomplished. As mentioned above, by further selecting the bias $\mathrm{H}$-field at $-80 \mathrm{Oe}$ where there exists only one magnetization distribution at each E-field ranging from $0-6 \mathrm{kV} \mathrm{cm}^{-1}$, reversible electrical modulation is also achieved.

Fig. $3 \mathrm{~g}$ shows the $\mathrm{M}-\mathrm{H}$ loops at 0 and $6 \mathrm{kV} \mathrm{cm}^{-1}$, with only one $M / M_{\mathrm{s}}$ value at $-80 \mathrm{Oe}$ under both 0 and $6 \mathrm{kV} \mathrm{cm}^{-1}$, that is, -0.23 at $0 \mathrm{kV} \mathrm{cm}^{-1}$ and 0.65 at $6 \mathrm{kV} \mathrm{cm}^{-1}$. Therefore, under the electrical modulation, the variation route of the $M / M_{\mathrm{s}}$ values is a closed circle that the $M / M_{\mathrm{s}}$ values can only vary between -0.23 and 0.65 , corresponding to the transverse components of magnetic domains changing between $225^{\circ}$ and $45^{\circ}$, resulting in the reversible control of the magnetization reversal by E-fields without additional $\mathrm{H}$-fields applied. In addition, the range of the bias $\mathrm{H}$-field to achieve reversible electrical control of magnetization reversal is from -100 to -70 Oe.

In order to further reduce the energy dissipation, electrical modulation of magnetization reversal is desired to be acquired without the external $\mathrm{H}$-field. Therefore, an appropriate $H_{\text {anneal }}$ deviating from the [01-1] direction is utilized to obtain the initial magnetization under $0 \mathrm{kV} \mathrm{cm}^{-1}$ opposite to that under Efields at 0 Oe. As shown in Fig. $4 \mathrm{a}$, if $H_{\text {anneal }}$ is close to the [100] direction with an angle a little larger than $90^{\circ}$ relative to the [01-1] direction, electrical modulation of magnetization reversal can be achieved with no external $\mathrm{H}$-field applied, where the transverse components of domains reverse between $60^{\circ}$ and $240^{\circ}$ at $\theta=60^{\circ}$. And with the $H_{\text {anneal }}$ in the opposite direction, the transverse components can also be driven to reverse by E-fields. The heterostructures were annealed with $H_{\text {anneal }}$ at $-15^{\circ}$ as an example.

Fig. $4 \mathrm{~b}$ illustrates the diagram of test direction and E-fields for electrical control of magnetism, where the white region separates areas with $M_{\mathrm{r}} / M_{\mathrm{s}}$ in positive or negative. There exists a wide window of test direction to realize the magnetization reversal by E-fields, roughly from $\theta=35^{\circ}$ to $\theta=70^{\circ}$. At $\theta=60^{\circ}$, the $M_{\mathrm{r}} / M_{\mathrm{s}}$ value can be driven to switch repeatedly between 0.32 at $0 \mathrm{kV} \mathrm{cm}^{-1}$ and -0.52 at $8 \mathrm{kV} \mathrm{cm}^{-1}$, only by changing the E-fields.

Meanwhile, vector imaging of electrical control of magnetization reversal at 0 Oe for $\theta=60^{\circ}$ is illustrated in Fig. $4 \mathrm{c}$. At $E_{0}=$ $0 \mathrm{kV} \mathrm{cm}^{-1}$, magnetic domains are mainly lying in $335^{\circ}-350^{\circ}$ with the transverse components along $60^{\circ}$. When the E-field is ascended to $E_{1}=8 \mathrm{kV} \mathrm{cm}^{-1}, 85 \%$ magnetic domains clockwise rotate along $292^{\circ}-330^{\circ}$ where the transverse components are in $240^{\circ}$. Thus, the electrical control of magnetization reversal can be realized with the transverse components of domains from $60^{\circ}$ to $240^{\circ}$ driven by E-fields. The pixel-by-pixel comparisons of domains between $E_{0}=0 \mathrm{kV} \mathrm{cm}{ }^{-1}$ and $E_{1}=8 \mathrm{kV} \mathrm{cm}^{-1}$ is shown in Fig. $4 \mathrm{~d}$, where $95 \%$ variation angles are located from $-10^{\circ}$ to $-50^{\circ}$. Fig. $4 \mathrm{e}$ shows that when the E-field is back to $E_{2}=$ $0 \mathrm{kV} \mathrm{cm}^{-1}$, magnetic domains substantially restore the initial magnetization distribution with transverse components along $60^{\circ}$, indicating that reversible control of magnetization reversal can be achieved at 0 Oe by altering the E-fields solely. With the increase of E-field to $E_{3}=8 \mathrm{kV} \mathrm{cm}^{-1}$, the clockwise rotation of magnetic domains results in the transverse component along $240^{\circ}$, which clarifies the reproducibility of electrical control of reversible magnetization reversal. Fig. $4 \mathrm{f}$ presents the variation angle of domains between $E_{2}=0 \mathrm{kV} \mathrm{cm}^{-1}$ and $E_{3}=8 \mathrm{kV} \mathrm{cm}^{-1}$, with $96 \%$ between $-10^{\circ}$ and $-50^{\circ}$. Furthermore, Figs S4 and S5 display the reversible electrical control of magnetization reversal under 0 Oe at $\theta=45^{\circ}$, where magnetic domains can be driven to rotate between $335^{\circ}-355^{\circ}$ and $295^{\circ}-315^{\circ}$, with variation angles from $-20^{\circ}$ to $-60^{\circ}$ over $90 \%$.

In addition, irreversible electrical control of magnetization reversal is also conducted as a supplement, which can be found in Figs S6-S11. For this case, during the deposition of the exchange-biased multilayers onto the PMN-PT(011) substrates, an $\mathrm{H}$-field $\left(H_{\text {grow }}\right)$ of $200 \mathrm{Oe}$ was applied along the [01-1] direction replacing the annealing, and the corresponding magnetic domains patterns at $\theta=45^{\circ}$ is presented in Fig. S6. Figs S7 and S8 illustrate dynamic magnetization processes under different E-fields. Furthermore, to realize the vector imaging of magnetization reversal by E-fields, magnetic domains at $-14 \mathrm{Oe}$ under $6 \mathrm{kV} \mathrm{cm}^{-1}$ are selected as the initial state, whose magnetization distribution can remain unchanged within $5 \mathrm{~min}$, as shown in Fig. S9. Fig. S10 illustrates that when the E-field is decreased to $0 \mathrm{kV} \mathrm{cm}^{-1}$, magnetic domains change obviously where a complex multi-domain state mainly along $180^{\circ}-225^{\circ}$ replaces that with domains in $90^{\circ}$, achieving the electrical modulation of magnetization reversal. As the E-field is increased to $6 \mathrm{kV} \mathrm{cm}^{-1}$ once more, the magnetization distribution cannot recover the initial state along $90^{\circ}$, but is mainly around $225^{\circ}-$ $255^{\circ}$. As a consequence, once the E-field is removed again, domains are driven to near $180^{\circ}-225^{\circ}$, that is, the magnetization reversal by E-field is irreversible. In order to achieve the reversible regulation, an additional $\mathrm{H}$-field is introduced to reproduce the initial state of electrical manipulation with magnetic 

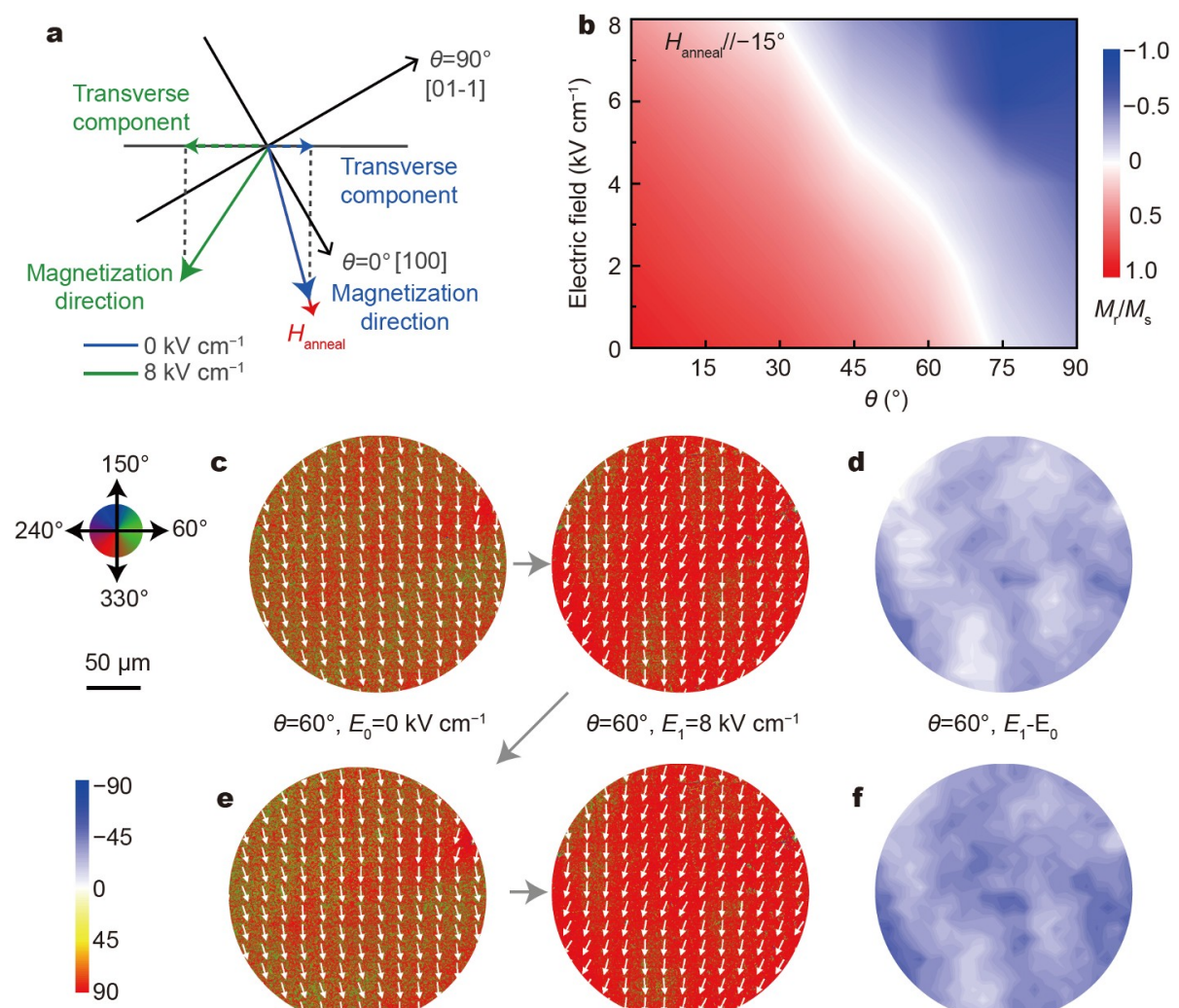

Angle variation $\left(^{\circ}\right)$
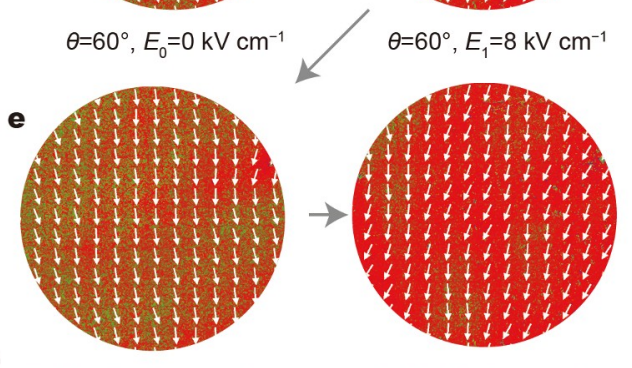

$\theta=60^{\circ}, E_{1}-E_{0}$

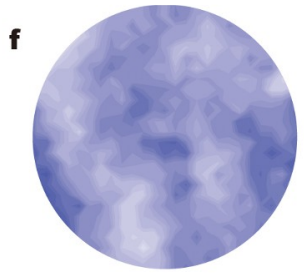

$\theta=60^{\circ}, E_{2}=0 \mathrm{kV} \mathrm{cm}^{-1}$

$\theta=60^{\circ}, E_{3}=8 \mathrm{kV} \mathrm{cm}^{-1}$

$\theta=60^{\circ}, E_{3}-E_{2}$

Figure 4 Reversible electrical control of magnetization reversal with no bias $\mathrm{H}$-fields. (a) Schematic of magnetization reversal induced by E-fields. (b) Diagram of $\theta$ and E-fields with $H_{\text {anneal }}$ at $-15^{\circ}$. (c) Magnetization reversal driven by E-fields at $\theta=60^{\circ}$. (d) Variation angles of domains via the pixel-bypixel comparisons. (e, f) The reversibility and reproducibility of electrical control of magnetization reversal for magnetic domain evolution (e), and variation angles (f).

domains along $90^{\circ}$, as shown in Fig. S11.

\section{CONCLUSIONS}

In summary, the vector imaging of reversible electrical control of magnetization reversal was demonstrated in exchange-biased $\mathrm{CoFeB} / \mathrm{IrMn} / \mathrm{NiFe} / \mathrm{PMN}-\mathrm{PT}(011)$ multiferroic heterostructures via in-situ quantitative MOKE. With the increase of E-fields from 0 to $6 \mathrm{kV} \mathrm{cm}^{-1}$ at $-80 \mathrm{Oe}$, the magnetization directions of magnetic domains were driven clockwise rotating from approximately $160^{\circ}$ to $80^{\circ}-120^{\circ}$, while the transverse components of domains switched from $225^{\circ}$ to $45^{\circ}$ with the magnetization reversal. Meanwhile, the reversibility of electrical modulation of magnetization reversal was achieved due to the recovered magnetization distribution just removing the E-fields with the $\mathrm{H}$-field maintained. Pixel-by-pixel comparisons of magnetic domains were performed to further clarify the reversibility and reproducibility of the E-field-driven magnetization switching. Furthermore, as the electrical regulation of magnetization reversal was achieved by the competition among various anisotropy energies, it could be realized with no bias $\mathrm{H}$-field via selecting suitable $H_{\text {anneal }}$. Vector imaging of electrical control in exchange-biased multiferroic systems could be useful for the development of energy-efficient spintronic devices involving exchange bias.

Received 24 March 2021; accepted 18 May 2021; published online 4 August 2021
1 Chappert C, Fert A, Van Dau FN. The emergence of spin electronics in data storage. Nat Mater, 2007, 6: 813-823

2 Son JY, Kim CH, Cho JH, et al. Self-formed exchange bias of switchable conducting filaments in $\mathrm{NiO}$ resistive random access memory capacitors. ACS Nano, 2010, 4: 3288-3292

3 Guo Y, Ouyang Y, Sato N, et al. Exchange-biased anisotropic magnetoresistive field sensor. IEEE Sens J, 2017, 17: 3309-3315

4 Röbisch V, Yarar E, Urs NO, et al. Exchange biased magnetoelectric composites for magnetic field sensor application by frequency conversion. J Appl Phys, 2015, 117: 17B513

5 Bibes M, Barthélémy A. Towards a magnetoelectric memory. Nat Mater, 2008, 7: 425-426

6 Wang L, Hu Z, Zhu Y, et al. Electric field-tunable giant magnetoresistance (GMR) sensor with enhanced linear range. ACS Appl Mater Interfaces, 2020, 12: 8855-8861

7 Zhang X, Zhan Q, Dai G, et al. Effect of mechanical strain on magnetic properties of flexible exchange biased $\mathrm{FeGa} / \mathrm{IrMn}$ heterostructures. Appl Phys Lett, 2013, 102: 022412

8 Reermann J, Durdaut P, Salzer S, et al. Evaluation of magnetoelectric sensor systems for cardiological applications. Measurement, 2018, 116: 230-238

9 Friedrich RM, Zabel S, Galka A, et al. Magnetic particle mapping using magnetoelectric sensors as an imaging modality. Sci Rep, 2019, 9: 2086

10 Song C, Cui B, Li F, et al. Recent progress in voltage control of magnetism: Materials, mechanisms, and performance. Prog Mater Sci, 2017, 87: $33-82$

11 Lage E, Kirchhof C, Hrkac V, et al. Exchange biasing of magnetoelectric composites. Nat Mater, 2012, 11: 523-529

12 Wu SM, Cybart SA, Yi D, et al. Full electric control of exchange bias. 
Phys Rev Lett, 2013, 110: 067202

13 Matsukura F, Tokura Y, Ohno H. Control of magnetism by electric fields. Nat Nanotech, 2015, 10: 209-220

14 Vaz CAF, Hoffman J, Ahn $\mathrm{CH}$, et al. Magnetoelectric coupling effects in multiferroic complex oxide composite structures. Adv Mater, 2010, 22: $2900-2918$

15 Zhang YJ, Chen JH, Li LL, et al. Ferroelectric strain modulation of antiferromagnetic moments in $\mathrm{Ni} / \mathrm{NiO}$ ferromagnet/antiferromagnet heterostructures. Phys Rev B, 2017, 95: 174420

16 He X, Wang Y, Wu N, et al. Robust isothermal electric control of exchange bias at room temperature. Nat Mater, 2010, 9: 579-585

17 Wu SM, Cybart SA, Yu P, et al. Reversible electric control of exchange bias in a multiferroic field-effect device. Nat Mater, 2010, 9: 756-761

18 Wei L, Hu Z, Du G, et al. Full electric control of exchange bias at room temperature by resistive switching. Adv Mater, 2018, 30: 1801885

19 Skumryev V, Laukhin V, Fina I, et al. Magnetization reversal by electric-field decoupling of magnetic and ferroelectric domain walls in multiferroic-based heterostructures. Phys Rev Lett, 2011, 106: 057206

20 Zhai K, Chai Y, Cong J, et al. Electric control of exchange bias in multiferroic hexaferrite $\mathrm{Ba}_{0.4} \mathrm{Sr}_{1.6} \mathrm{Mg}_{2} \mathrm{Fe}_{12} \mathrm{O}_{22}$. Phys Rev B, 2018, 98: 144405

21 Liu M, Lou J, Li S, et al. E-field control of exchange bias and deterministic magnetization switching in AFM/FM/FE multiferroic heterostructures. Adv Funct Mater, 2011, 21: 2593-2598

22 Chen A, Zhao Y, Li P, et al. Angular dependence of exchange bias and magnetization reversal controlled by electric-field-induced competing anisotropies. Adv Mater, 2016, 28: 363-369

23 Lahtinen THE, Tuomi JO, van Dijken S. Pattern transfer and electricfield-induced magnetic domain formation in multiferroic heterostructures. Adv Mater, 2011, 23: 3187-3191

24 Zhong G, An F, Bitla Y, et al. Deterministic, reversible, and nonvolatile low-voltage writing of magnetic domains in epitaxial $\mathrm{BaTiO}_{3} / \mathrm{Fe}_{3} \mathrm{O}_{4}$ heterostructure. ACS Nano, 2018, 12: 9558-9567

25 Yao J, Song X, Gao X, et al. Electrically driven reversible magnetic rotation in nanoscale multiferroic heterostructures. ACS Nano, 2018, 12: $6767-6776$

26 Mikheev E, Stolichnov I, De Ranieri E, et al. Magnetic domain wall propagation under ferroelectric control. Phys Rev B, 2012, 86: 235130

27 Li P, Zhao Y, Zhang S, et al. Spatially resolved ferroelectric domainswitching-controlled magnetism in $\mathrm{Co}_{40} \mathrm{Fe}_{40} \mathrm{~B}_{20} / \mathrm{Pb}\left(\mathrm{Mg}_{1 / 3} \mathrm{Nb}_{2 / 3}\right)_{0.7} \mathrm{Ti}_{0.3} \mathrm{O}_{3}$ multiferroic heterostructure. ACS Appl Mater Interfaces, 2017, 9: 26422649

28 Ba Y, Liu Y, Li P, et al. Spatially resolved electric-field manipulation of magnetism for $\mathrm{CoFeB}$ mesoscopic discs on ferroelectrics. Adv Funct Mater, 2018, 28: 1706448

29 Ghidini M, Mansell R, Maccherozzi F, et al. Shear-strain-mediated magnetoelectric effects revealed by imaging. Nat Mater, 2019, 18: 840845

30 Liu M, Nan T, Hu JM, et al. Electrically controlled non-volatile switching of magnetism in multiferroic heterostructures via engineered ferroelastic domain states. NPG Asia Mater, 2016, 8: e316

31 Franke KJ, Van de Wiele B, Shirahata Y, et al. Reversible electric-fielddriven magnetic domain-wall motion. Phys Rev X, 2015, 5: 011010

32 Chung TK, Carman GP, Mohanchandra KP. Reversible magnetic domain-wall motion under an electric field in a magnetoelectric thin film. Appl Phys Lett, 2008, 92: 112509

33 Schellekens AJ, van den Brink A, Franken JH, et al. Electric-field control of domain wall motion in perpendicularly magnetized materials. Nat Commun, 2012, 3: 847

34 Koyama T, Nakatani Y, Ieda J', et al. Electric field control of magnetic domain wall motion via modulation of the dzyaloshinskii-moriya interaction. Sci Adv, 2018, 4: eaav0265

Acknowledgements This work was supported by the National Key R\&D Program of China (2018YFB0407601), the National Natural Science Foundation of China (91964109, 62071374 and 51802248), and the National 111 Project of China (B14040).
Author contributions $\mathrm{Hu} \mathrm{Z}$, Pan JY and Zhao X conceived the project. Zhao $\mathrm{X}$ fabricated the samples and performed the measurements with assistant from Fang T, Cheng Y, Shi K, Weng YX, Du Y, Wu J and Guan M. Zhao $\mathrm{X}$ wrote the manuscript with the support from $\mathrm{Hu} Z$, Wang $\mathrm{Z}$, Zhou $\mathrm{Z}$, Liu M and Pan JY. All authors contributed to the general discussion.

Conflict of interest The authors declare that they have no conflict of interest.

Supplementary information Experimental details and supporting data are available in the online version of the paper.

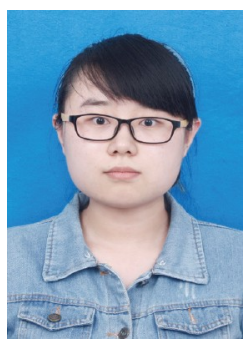

Xinger Zhao received the BS degree in applied physics from Inner Mongolia University, Hohhot, China, in 2016. She is currently working towards the $\mathrm{PhD}$ degree in electronic science and technology at Xi'an Jiaotong University, Xi'an, China. Her research interests include magnetoelectric materials and magnetic domain manipulation.

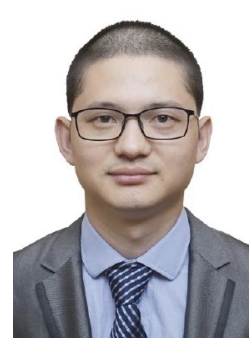

Zhongqiang $\mathbf{H u}$ is a professor of electrical engineering at Xi'an Jiaotong University. He received his $\mathrm{PhD}$ degree in microelectronics and solid state electronics from Wuhan University, China, in 2012. $\mathrm{He}$ did postdoctoral research at Argonne National Laboratory and Northeastern University from 2012 to 2015. His research focuses on magnetoelectric materials and devices, including magnetic sensors, magnetoresistive random access memory, and adjustable filters.

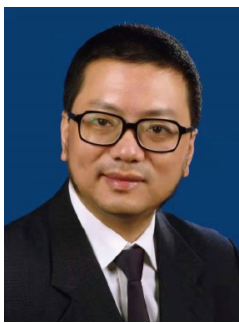

Jing-Ye Pan has been working as a doctor in intensive care unit, the First Affiliated Hospital of Wenzhou Medical University for 26 years. He is the academic leader of critical illness, and the director of the Key Laboratory of Intelligent Critical Care and Life Support Research of Zhejiang Province. His current research interests lie in interdisciplinary areas between flexible electronics and diseases.

\section{交换偏置多铁异质结中电场调控可逆性磁化反转的 矢量分析研究}

赵星儿 ${ }^{1}$, 胡忠强 ${ }^{*}$, 方婷 ${ }^{1}$, 程宇心 ${ }^{1}$, 施可庆 ${ }^{2}$, 翁一心 ${ }^{3}$, 杜泳君 ${ }^{1}$, 吴金根 ${ }^{1}$, 关蒙萌 ${ }^{1}$, 王志广 $广^{1}$, 周子尧 ${ }^{1}$, 刘明 ${ }^{1}$, 潘景业 ${ }^{3 *}$

摘要 铁磁与反铁磁层间的交换偏置效应已在自旋电子器件中得到了 广泛应用. 目前, 利用电场调控这一低能耗的方式已经实现了对交换偏 置磁性多层膜中如交换偏置场和磁化值等宏观性质的调节. 然而, 交换 偏置体系中磁畴如何随施加电场进行演化却鲜有报道. 本文采用定量 磁光克尔效应 (MOKE) 显微成像系统, 实现了原位矢量分析 $\mathrm{CoFeB} /$ IrMn/PMN-PT(011)交换偏置多铁异质结中电场作用下的可逆磁化反 转. 在保持偏置磁场于 $-80 \mathrm{Oe}$ 不变的情况下, 外加电场驱动磁畴的磁化 方向在 $160^{\circ}$ 方向与 $80^{\circ}-120^{\circ}$ 方向区间内往复变化, 对应磁畴的横向分量 也于 $225^{\circ}$ 方向与 $45^{\circ}$ 方向间来回切换. 同时, 通过逐像素比对不同电场下 的磁畴, 进一步证实了电场调节磁化反转的可逆性. 此外, 在零偏置磁 场下也实现了对磁化反转的可逆电场调节. 电场调控交换偏置的矢量 分析对于理解磁电效应以及发展下一代自旋电子器件具有重要意义. 